\title{
High resolution probe of coherence in low-energy charge exchange collisions with oriented targets
}

\author{
A. Leredde,${ }^{1}$ X. Fléchard, ${ }^{1}$ A. Cassimi,${ }^{2}$ D. Hennecart, ${ }^{2}$ and B. Pons ${ }^{3}$ \\ ${ }^{1}$ LPC Caen, ENSICAEN, Universit de Caen, CNRS/IN2P3, Caen, France \\ ${ }^{2}$ CIMAP, CEA - CNRS - ENSICAEN, BP 5133, F-14070, Caen cedex 5, France \\ ${ }^{3}$ CELIA, Univ. Bordeaux - CNRS - CEA, F-33405 Talence, France.
}

(Dated: July 30, 2018)

\begin{abstract}
The trapping lasers of a magneto-optical trap (MOT) are used to bring $\mathrm{Rb}$ atoms into well defined oriented states. Coupled to recoil-ion momentum spectroscopy (RIMS), this yields a unique MOTRIMS setup which is able to probe scattering dynamics, including their coherence features, with unprecedented resolution. This technique is applied to the low-energy charge exchange processes $\mathrm{Na}^{+}+\mathrm{Rb}\left(5 p_{ \pm 1}\right) \rightarrow \mathrm{Na}(3 p, 4 s)+\mathrm{Rb}^{+}$. The measurements reveal detailed features of the collisional interaction which are employed to improve the theoretical description. All of this enables to gauge the reliability of intuitive pictures predicting the most likely capture transitions.
\end{abstract}

PACS numbers: 34.10.+x,34.70.+e,37.10.Gh

Apprehending the dynamics of nonadiabatic processes in atomic and molecular physics is not an easy task. The initial and final states have generally complex fine structures and even in the case of well-defined boundary conditions, multiple pathways can drive the dynamics from the beginning to the end of the process. Beyond the identification of the reaction routes, their relative coherence must explicitely be considered since it monitors the eventual occurence of interferences and determines the final outcome of the reaction. The degree of coherence, that our (basically classical) intuition cannot easily grasp, is generally revealed by quantum mechanical or semiclassical calculations. However continuous advances in coincidence and probe techniques have opened the way to 'quantum mechanical complete' coherence experiments [1] which are able to measure not only the reaction probabilities but also the relative phase of the transition amplitudes. Such experiments have been performed for lowenergy ion-atom collisions [2-7], electron impact excitation of helium [8, 9] and photon-induced ionization of krypton atoms [10]. Nevertheless, in most of the cases involving heavy particle impact, the experimental resolution did not allow to scrutinize the details of the scattering patterns predicted theoretically.

We are interested in ion-atom collisions in the $\mathrm{keV}$ impact energy range where the most probable inelastic process is electron transfer from the target to the projectile 11]. We have recently demonstrated that the transfer dynamics in $\mathrm{Na}^{+}+\mathrm{Rb}(5 s, 5 p)$ collisions can be probed with unsurpassed accuracy by coupling cold-target recoil-ion spectroscopy (COLTRIMS [12]) to laser-cooled atomic targets trapped in a magneto-optical trap (MOT) 1315]. Here we show that the MOT trapping laser pulses can further be employed to bring the target into an oriented initial state, yielding a novel MOTRIMS setup which enables to picture the charge exchange dynamics, including its coherence features, with unprecedented resolution. We still consider $\mathrm{Na}^{+}+\mathrm{Rb}$ collisions, but optical

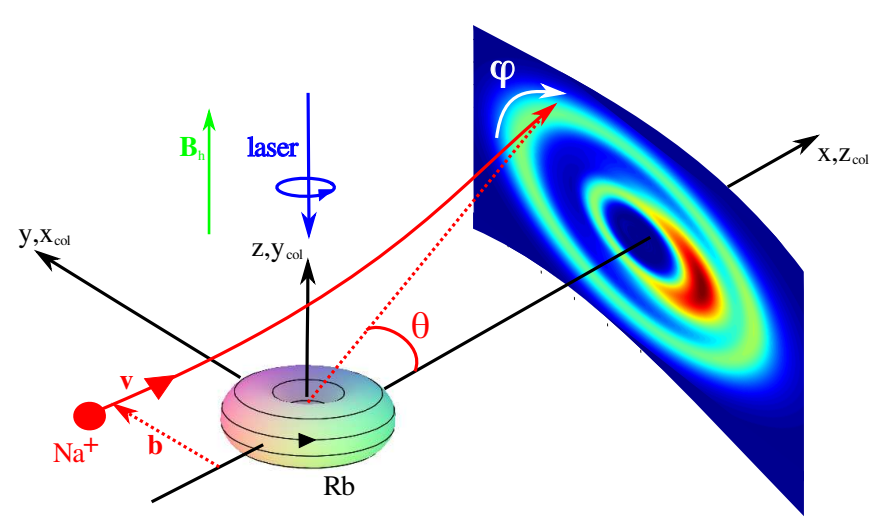

FIG. 1: A homogeneous magnetic field $B_{h}$ sets the quantification $z$-axis and optical pumping leads to magnetic sublevels of the target state with well defined hyperfine $m_{F}$ quantum numbers, depending on the handedness of the laser pulse. We illustrate this for the $\operatorname{Rb}\left(5^{2} P_{3 / 2}, F=3, m_{F}=+3\right) \equiv$ $\mathrm{Rb}\left(5 p_{+1}\right)$ case. $\mathrm{Na}^{+}$impinges on the oriented target impact with initial velocity $\mathbf{v}$ and impact parameter $\mathbf{b}$.

pumping from a circularly polarized laser pulse selects the entry state as $5 p_{ \pm 1}$. The high-resolution angular differential cross sections (DCS) are compared to molecular orbital close-coupling (MOCC) calculations performed in the framework of the single active electron (SAE) approximation [16]. This comparison reveals subtle features of the projectile-target interaction which monitors the state-selective scattering, and further allows us to mark out the accuracy of the widely used SAE approach. Additionally the DCS's show a pronounced and finely resolved asymmetry between left- and right-handed scatterings, related to the coherence of the charge-exchange process from the selected oriented state. These orientation effects are theoretically studied from both classical and quantum perspectives. 
Our MOTRIMS setup has been described in details elsewhere [16, 17]. We therefore focus hereinafter on the transformations we made to merge efficient orientation and RIMS techniques within a MOT.

The high resolution performances of our MOTRIMS setup rely on a transverse extraction of the recoil ions coupled to a fast switch-off of the trapping magnetic field during data counting [16]. The field-free requirement is hardly compatible with a definite orientation of the target states since weak parasite magnetic fields, including the terrestrial one, can randomly influence the state alignment which has to be first strictly fixed. The total kinetic momentum of the target is thus unambiguously aligned, for both the ground $\operatorname{Rb}\left(5^{2} S_{1 / 2}, F=2\right)$ and excited $\operatorname{Rb}\left(5^{2} P_{3 / 2}, F^{\prime}=3\right)$ states, by using a homogeneous magnetic field $B_{h}$ along the $z$-axis which is taken as the quantization direction (see Fig. 11). $B_{h}$ is provided by Helmotz coils, and its magnitude is 2 Gauss, which is large enough to depreciate any perturbing magnetic field. To compensate the action of $B_{h}$ during the trapping period, one of the MOT anti-Helmotz coil is shifted up by $4 \mathrm{~mm}$ so that the field-free requirement is obeyed at the center of the collision chamber.

To bring the atoms $\operatorname{Rb}\left(5^{2} S_{1 / 2}, F=2\right)$ and $\operatorname{Rb}\left(5^{2} P_{3 / 2}, F^{\prime}=3\right)$ into oriented $m_{F}=+2$ and $m_{F}^{\prime}=+3$ magnetic sublevels, respectively, we optically pump them using a left-handed circularly polarized laser beam, coming along the $-z$ direction, that induces $\sigma^{+}$transitions. The opposite orientation is obtained using a right-handed laser beam. The polarizing laser beam (PLB), coming from one of the trapping lasers, is diffracted by an AOM (Acousto-Optic Modulator) and tuned slightly to the red of the $\left(5^{2} S_{1 / 2}, F=2\right) \rightarrow\left(5^{2} P_{3 / 2}, F^{\prime}=3\right)$ transition. This AOM can be switched on and off to control the status of the PLB. A half-wave plate coupled to a PBSC (Polarizing Beam Splitting Cube) provides a linearly polarized beam with tunable intensity. The beam is circularly polarized using a quarter-wave plate and directed towards the target. A counter propagating laser beam, obtained by a retro-reflection at the exit side of the collision chamber, reduces net momentum transfer from the PLB to the target atoms. In spite of this retroreflected beam, the cold cloud is still warmed up and lost after a few ms with a time constant depending on the intensities and alignment of the laser beams. An incoming laser beam with a power of $1 \mathrm{~mW} / \mathrm{cm}^{2}$ was thus used to provide a target with a large excited fraction without pushing the cold atomic cloud outside of the collision region.

The polarization speed and orientation yield have been characterized by means of absorption and fluorescence techniques, respectively. We found that more than $95 \%$ of the atoms are conveniently oriented within a time interval shorter than $5 \mu \mathrm{s}$. Experiments were performed with oriented $\operatorname{Rb}\left(5^{2} P_{3 / 2}, F=3, m_{F}= \pm 3\right) \equiv \operatorname{Rb}\left(5 p_{ \pm 1}\right)$ targets at 5,2 and $1 \mathrm{keV}$. Acquisition times for both
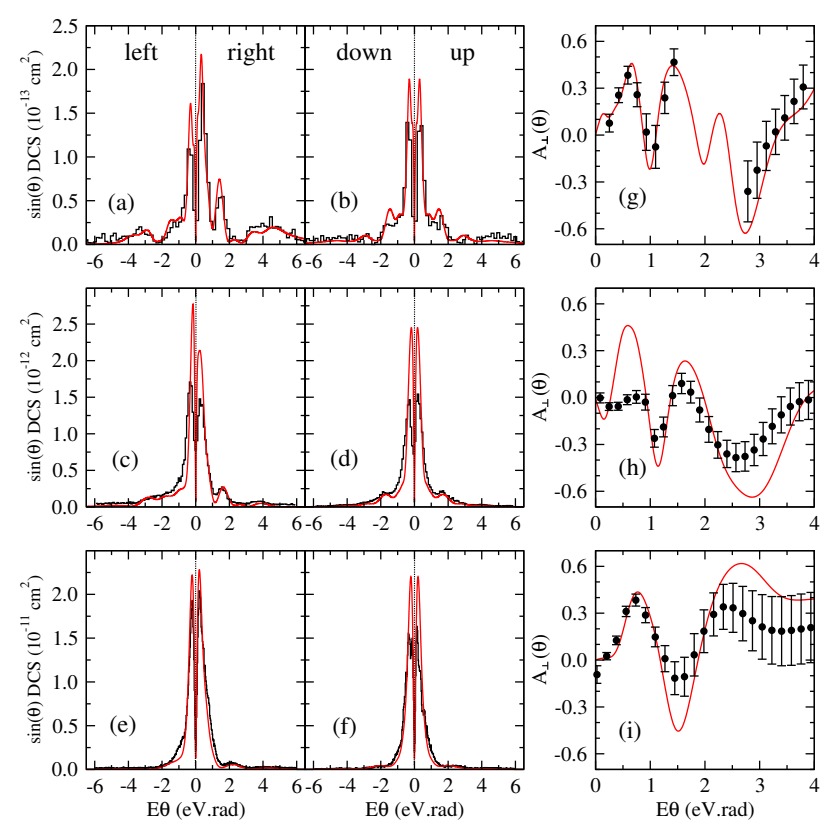

FIG. 2: Weighted DCS's relative to the charge-exchange reaction $\mathrm{Na}^{+}+\mathrm{Rb}\left(5 p_{+1}\right) \rightarrow \mathrm{Na}(3 p)+\mathrm{Rb}^{+}$at $E=1(\mathrm{a}-\mathrm{b}), 2$ (c-d) and 5 (e-f) $\mathrm{keV}$, as functions of $E \theta$. The histograms refer to the measurements while the continuous (red) lines correspond to MOCC calculations. In (g-i) are displayed the left-right coherence parameters $A_{\perp}(\theta)$ defined in (2) for $E=1,2$ and 5 $\mathrm{keV}$ respectively.

right- and left-handed orientations of the target were of about 24 hours for each energy. We present in Fig. 2(a-f) the DCS associated to the main $\mathrm{Na}^{+}+\mathrm{Rb}\left(5 p_{+1}\right)$ $\rightarrow \mathrm{Na}(3 p)+\mathrm{Rb}^{+}$charge-exchange reaction. The angular resolution achieved ranges from $\Delta \theta=70 \mu \mathrm{rad}$ at $1 \mathrm{keV}$ to $28 \mu \mathrm{rad}$ at $5 \mathrm{keV}$. The measurements are compared to MOCC calculations detailed in [16]. Briefly MOCC is a semiclassical approach which combines a classical description of nuclear motion and a quantum-mechanical description of electron transitions. Only nonadiabatic transitions involving the $\mathrm{Rb}$ valence electron are considered and both the target and projectile atomic cores are assumed to remain frozen throughout the collision. Parametric model potentials then represent the interactions of the active electron with the ionic cores [16], and the description of the core-core interaction is first restricted to its coulombic repulsive part, as usual in treatments of collisions with dressed target and projectile (see e.g. [18, 19]). MOCC employs real orbitals $\left\{p_{x}, p_{y}, p_{z}\right\}$ and computes the scattering amplitudes $T_{p_{x, y, z} \rightarrow p_{x, y, z}}^{c o l}(\theta)$ in the collisional frame $\left(x_{c o l}, y_{c o l}, z_{c o l}\right)$ where the $z_{c o l}$ and $x_{c o l}$-axis are defined by the projectile beam and the impact parameter directions respectively. The amplitudes $T^{c o l}$ are then rotated to correspond to initial and final oriented states defined in the photonic reference frame $(x, y, z)$, as documented in e.g. [19, 20] and illustrated in 
Fig. 1. This yields

$$
\begin{array}{r}
T_{p_{+1} \rightarrow p_{ \pm 1}}(\theta, \varphi)=\mp \frac{1}{2} T_{p_{z} \rightarrow p_{z}}^{c o l}-\frac{1}{2} \cos ^{2}(\varphi) T_{p_{x} \rightarrow p_{x}}^{c o l} \\
+\frac{1}{2} \sin ^{2}(\varphi) T_{p_{y} \rightarrow p_{y}}^{c o l}+\frac{i}{2} \cos (\varphi)\left[T_{p_{z} \rightarrow p_{x}}^{c o l} \mp T_{p_{x} \rightarrow p_{z}}^{c o l}\right] \\
T_{p_{+1} \rightarrow p_{0}}(\theta, \varphi)=\frac{\sqrt{2}}{2} \sin (\varphi) T_{p_{z} \rightarrow p_{x}}^{c o l}+\frac{i \sqrt{2}}{4} \sin (2 \varphi) \\
{\left[T_{p_{x} \rightarrow p_{x}}^{c o l}+T_{p_{y} \rightarrow p_{y}}^{c o l}\right]}
\end{array}
$$

We do not resolve experimentally the $m$ contributions to the $3 p$ DCS and therefore display in Fig. 2(a-f) the weighted DCS $\sin (\theta) \sigma_{p_{+1} \rightarrow 3 p}=$ $\sin (\theta)\left(\left|T_{p_{+1} \rightarrow p_{+1}}\right|^{2}+\left|T_{p_{+1} \rightarrow p_{0}}\right|^{2}+\left|T_{p_{+1} \rightarrow p_{-1}}\right|^{2}\right)$. Furthermore, for sake of quantitative comparison between experiments and theory, the DCS is presented in terms of its four main contributions to which we refer to as left $(\varphi=0)$, up $(\varphi=\pi / 2)$, right $(\varphi=\pi)$ and down $(\varphi=3 \pi / 2)$ [21]. The calculations are convoluted according to the experimental resolution $\Delta \theta$ previously detailed.

The agreement of experimental and computed DCS's is very good, and this is even more satisfactory as we normalize only the whole measured signal to the total computed cross section. Because of the reflexion symmetry with respect to the $(x, y)$-plane, the up and down contributions are identical. This is not the case for the left and right contributions: the rotation of the electron flow inherent in the initial oriented state breaks the symmetry of left $(y>0)$ and right $(y<0)$ scatterings (see Fig. 1). The left/(right+left) total ratios, $R$, derived from the measurements and MOCC calculations are in striking agreement; for instance, $R^{M O T R I M S}=$ $37.7 \%$ and $R^{M O C C}=37.5 \%$ at $E=1 \mathrm{keV}$. Furthermore, and importantly, the exceptional angular resolution reached by our improved setup $(\Delta \theta \sim 70 \mu \mathrm{rad}$ at $1 \mathrm{keV}$ ) enables to resolve the oscillatory patterns of the DCS and associated ( $\theta$-dependent) left-right asymmetry. This would have been clearly impossible with conventional setups which rather deal with $\Delta \theta \sim 1$ mrad. The $\theta$-dependent left-right asymmetry, defined as $A_{\perp}(\theta)=\frac{\sigma_{p_{+1} \rightarrow 3 p}(\theta, 0)-\sigma_{p_{+1} \rightarrow 3 p}(\theta, \pi)}{\sigma_{p_{+1} \rightarrow 3 p}(\theta, 0)+\sigma_{p_{+1} \rightarrow 3 p}(\theta, \pi)}$, is nothing else but a direct measure of the importance of interference effects between 'radial' $\left(p_{z} \rightarrow p_{z}, p_{x} \rightarrow p_{x}\right)$ and 'rotational' $\left(p_{z} \rightarrow p_{x}, p_{x} \rightarrow p_{z}\right)$ collisional transition pathways since one obtains from equations (11)

$$
A_{\perp}(\theta)=\frac{2 \operatorname{Im}\left(T_{p_{z} \rightarrow p_{z}} T_{p_{x} \rightarrow p_{z}}^{*}-T_{p_{x} \rightarrow p_{x}} T_{p_{z} \rightarrow p_{z}}^{*}\right)}{\left|T_{p_{z} \rightarrow p_{z}}\right|^{2}+\left|T_{p_{x} \rightarrow p_{x}}\right|^{2}+\left|T_{p_{z} \rightarrow p_{x}}\right|^{2}+\left|T_{p_{x} \rightarrow p_{z}}\right|^{2}}
$$

In other words, $A_{\perp}(\theta)$ gives direct access to the degree of coherence of the $\mathrm{Rb}\left(5 p_{+1}\right) \rightarrow \mathrm{Na}(3 p)$ charge exchange mechanism. $A_{\perp}(\theta)$ is presented in Fig. 2(g-i) for $E=1,2$ and $5 \mathrm{keV}$. At $E=1 \mathrm{keV}, A_{\perp}(\theta)$ could not be safely derived from the measurements between $E \theta=1.5$ and 2.5 eV.rad because of excessively noisy left and right signals.

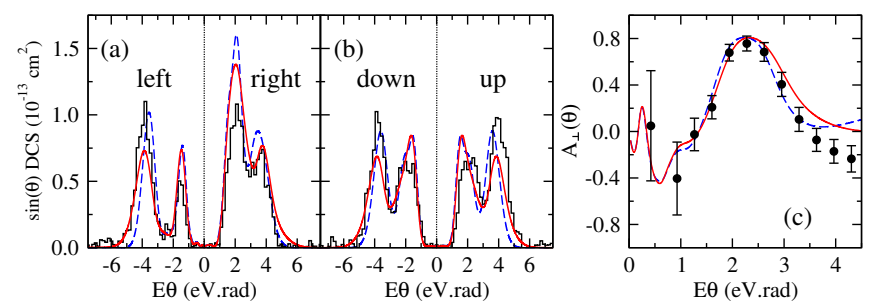

FIG. 3: Same as Fig. 2 for $\mathrm{Na}^{+}+\mathrm{Rb}\left(5 p_{+1}\right) \rightarrow \mathrm{Na}(4 s)+\mathrm{Rb}^{+}$ at $E=1 \mathrm{keV}$. The dashed (blue) and continuous (red) lines correspond to MOCC calculations accounting or not for highorder multipole core-core interactions.

The overall agreement of the measured and predicted coherence pictures is satisfactory. As $E$ decreases, the interferential pattern presents more structures within a fixed $E \theta$ range which, according to the classical description of scattering, should mark the range of involved impact parameters $b$ through $b=1 / E \theta[11]$. This behaviour stems from the fact that the phases of the collisional transition amplitudes are inversely proportional to the impact velocity $v$ [16], which enhances the number of constructive and destructive occurences in the $T$-cross products of (2) within a fixed $b$-range as $E$ decreases.

In spite of the rather low statistics inherent in $(\theta, \varphi)$ differential measurements, our setup has allowed us to reliably extract from the raw data the signal associated to the secondary capture channel $\mathrm{Na}^{+}+\mathrm{Rb}\left(5 p_{+1}\right)$ $\rightarrow \mathrm{Na}(4 s)+\mathrm{Rb}^{+}$at $E=1 \mathrm{keV}$. To our knowledge no previous measurements were able to scrutinize secondary transfer dynamics at the coherence level. The DCS and corresponding $A_{\perp}(\theta)$ are presented in Fig. 3. MOCC and MOTRIMS lead to a double ring structure of the DCS which is mainly imprinted by $5 p_{0} \rightarrow 4 s$ transitions occuring at rather small internuclear distances $R<12$ u.a. But while the measurements yield a maximum outer ring, MOCC leads to the opposite. We demonstrate in Fig. 3 that this is due to the inadequate representation of the core-core interaction in terms of purely net coulombic repulsion $1 / R$. We have indeed implemented improved MOCC calculations in which the mutual polarization of the cores is accounted by the attractive ion-dipole $-\left(\alpha_{d}^{R b^{+}}+\alpha_{d}^{N a^{+}}\right) / 2 R^{4}$ and dipole-dipole $-\left(\alpha_{q}^{R b^{+}}+\alpha_{q}^{N a^{+}}\right) / 2 R^{6}$ potentials, where $\alpha_{d}$ and $\alpha_{q}$ are the dipole and quadrupole polarizabilities of the ionic cores 22]. These additional terms are naturally cancelled at small $R$, where the total core-core interaction must be repulsive, since we introduce in our MOCC calculations a cut-off function which prevents the system from entering the $R$-domain where the cores overlap [16]. It is clear in Fig. [3(a,b) that accounting for high multipole terms in the core-core interaction significantly affects the $4 s$ scattering and improves the agreement of calculations and measurements. In the case of capture into the $3 p$ shell, which mostly occurs at large $R(R \gtrsim 15$ a.u.), changes 


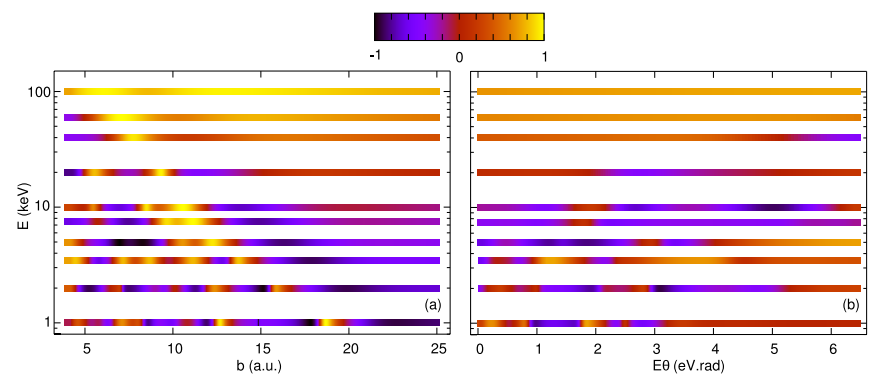

FIG. 4: (a) Classical $P_{ \pm 1}^{c}(b)$ and (b) quantum mechanical $P_{ \pm 1}^{q m}(\theta)$ orientation parameters for $5 p_{+1} \rightarrow 3 p$ right-hand capture scattering, for selected impact energies $E$.

with respect to the net coulombic behaviour were found to be small.

The asymmetry parameter associated to charge exchange into $4 s$ directly measures the inteferences between radial $p_{z} \rightarrow s$ and rotational $p_{x} \rightarrow s$ pathways, since $A_{\perp}(\theta)=2 \operatorname{Im}\left(T_{p_{z} \rightarrow s} T_{p_{x} \rightarrow s}^{*}\right)$. The improved MOCC calculations enhance the agreement of the computed $A_{\perp}$ with its experimental counterpart between $E \theta=2$ and 3 eV.rad. Beyond this range, both results deviate. In fact the dipolar and quadrupolar core-core potentials introduced in the improved calculations induce strong oscillating phases in the expression of the scattering amplitudes $T$. These phases, which respectively behave as $1 /\left(v b^{3}\right)$ and $1 /\left(v b^{5}\right)$, are essential to correctly reproduce the relative height of the DCS maxima as previously observed, but erode the outer part of the DCS upon integration on $b$. This results in a slight mismatch of the positions of the outer DCS maxima and leads to the deviation of $A_{\perp}$ at large $\theta$ 's. Improving further the theoretical calculations is beyond our present capabilities for such a complex system as $\mathrm{Na}^{+}+\mathrm{Rb}$ : prohibitive ab initio calculations should be performed to explicitely represent the molecularization of the ionic cores at small $R$, well beyond the present model potential with frozen atomic cores SAE description. This notwithstanding, the present improved MOCC calculations show a whole satisfactory agreement with experiments.

Even though we do not resolve experimentally the $m$ contributions to a final $(l, m)$ capture channel, we can take adavantage of the reliability of the MOCC calculations previously established to gauge how simple and intuitive pictures of orientation effects hold in the present low-energy charge exchange scattering. A first (and basically classical) picture is based on a velocity-matching model which predicts that capture preferentially occurs when the current flow of the target's valence electron is concurring with the velocity direction of the passing ion [26, 27]. Starting from $\operatorname{Rb}\left(5 p_{+1}\right)$, this would mean that right-hand collisions should be favoured and produce $\mathrm{Na}\left(3 p_{+1}\right)$ within the $3 p$ shell - see Fig. 11 An alternative picture relies on the analysis of the phases entering the quantum mechanical scattering amplitudes and prescribes that capture preferentially occurs if stationarity of the phases is possible upon integration on $b$. This gave rise to the so-called propensity rules [28, 29] which would give advantage to the $\mathrm{Rb}\left(5 p_{+1}\right) \rightarrow \mathrm{Na}\left(3 p_{-1}\right)$ capture process for right-hand collisions. In order to discriminate between these (contradictory) predictions, we compute the state-resolved orientation parameter

$$
P_{ \pm 1}^{q m}(\theta)=\frac{\left|T_{p_{+1} \rightarrow p_{+1}}(\theta, \pi)\right|^{2}-\left|T_{p_{+1} \rightarrow p_{-1}}(\theta, \pi)\right|^{2}}{\left|T_{p_{+1} \rightarrow p_{+1}}(\theta, \pi)\right|^{2}+\left|T_{p_{+1} \rightarrow p_{-1}}(\theta, \pi)\right|^{2}}
$$

which is essentially quantum mechanical in the sense that the scattering amplitudes $T$ underly integration on $b$ and therefore allow for interferences between classical trajectories with distinct $b$ 's [29]. A classical counterpart to $P_{ \pm 1}^{q m}, P_{ \pm 1}^{c}$, can be derived by replacing the scattering amplitudes $T$ in (3) by the transition amplitudes $\left\langle\phi_{ \pm 1} \mid \Psi\right\rangle$ issued from the MOCC calculations, where $\Psi$ is the total wavefunction at the end of the collision with fixed $v$ and $b$ and $\mid \phi_{ \pm 1}>$ are the $\mathrm{Na}\left(3 p_{ \pm 1}\right)$ oriented final states. $P_{ \pm 1}^{c}(b)$ is computed independently for each $b$, it does not allow trajectory interplay. $P_{ \pm 1}^{q m}(\theta)$ and $P_{ \pm 1}^{c}(b)$ are represented in Fig. (4) for various impact energies ranging from 1 to $100 \mathrm{keV}$. For $E<20 \mathrm{keV}$, our results show that neither the velocity matching nor the propensity rules, which would respectively lead to $P_{ \pm 1}=+1$ and -1 , apply. In this regime, the impact velocity is low enough to let the electron adapt almost adiabatically to the nuclear motion so that the velocity matching criterion is not decisive. On the other hand, it is known that stationarity does not drastically determine the propensity in singly-charged systems [29]. However, as $E$ increases, $v$ approaches the target's valence electron velocity, and it is clearly seen from both Figs. 4(a,b) that the velocity matching prevails. This is quite satisfying as this last model draws near our intuition of the charge exchange dynamics. Nevertheless we reiterate the conclusions of 29]: care must be taken when examining orientation effects and related dynamics from the classical perspective at low $E ; P_{ \pm 1}^{q m}(\theta)$ and $P_{ \pm 1}^{c}(b)$ cannot be linked quantitatively through the $E \theta \leftrightarrow 1 / b$ correspondence previously mentioned, as revealed by the comparison of Figs 4(a) and (b). Nuclear trajectory interferences blur this correspondence.

To sum up, a MOTRIMS setup have been modified in order to address the case of low-energy atomic collisions with oriented targets. Asymmetry in the DCS's, and related coherence properties, have then been observed with unprecedented resolution, not only for the main but also for secondary charge exchange processes. The measurements have revealed the importance of high-order projectile-target interactions, beyond the usual Coulombian description. This has enabled to improve the theoretical description but also marked out the limits of the SAE and frozen core approximations. We further have shown that none of the intuitive pictures of electron transfer dynamics apply at low impact energies, even 
though the velocity matching prevails as the impact velocity approaches the target electron's one. Finally, we want to emphasize that the improved MOTRIMS protocol is amenable to other scattering dynamics and particle impact. We venture that such applications would enhance the experimental/theoretical synergy in many other fields than the prototypical electron capture case herein observed.

[1] N. Andersen, J. W. Gallagher and I. V. Hertel, Phys. Rep. 165, 1 (1988).

[2] J. W. Thomsen et al., J. Phys. B 29, 5459 (1996).

[3] Z. Roller-Lutz et al., J. Phys. B 26, 2697 (1993).

[4] J. Salgado et al., J. Phys. B 30, 3059 (1997).

[5] J. W. Thomsen et al., J. Phys. B 32, 5189 (1999).

[6] Z. Roller-Lutz et al., Phys. Rev. A. 61, 022710 (2000).

[7] D. Dowek et al., J. Phys. B 35, 2051 (2002).

[8] A. G. Mikosza, J. F. Williams and J. B. Wang, Phys. Rev. Lett. 79, 3375 (1997).

[9] D. Cvejanovic, D. T. McLaughlin and A. Crowe, J. Phys. 33, 3013 (2000).

[10] E. Goulielmakis et al., Nature 466, 739 (2010).

[11] B. H. Bransden and M. H. C. McDowell, Charge Exchange and the Theory of Ion-Atom Collisions (Clarendon, Oxford, 1992).

[12] R. Dörner et al., Phys. Rep. 330, 95 (2000).
[13] M. van der Poel et al., Phys. Rev. Lett. 87, 123201 (2001).

[14] J. W. Turkstra et al., Phys. Rev. Lett. 87, 123202 (2001).

[15] X. Fléchard et al., Phys. Rev. Lett. 87, 123203 (2001).

[16] A. Leredde et al, Phys. Rev. A. 83, 1131 (2012).

[17] J. Blieck et al, Rev. Sci. Instrum. 79, 1 (2008).

[18] A. Dubois, S. E. Nielsen and J. P. Hansen, J. Phys. B 26, 705 (1993).

[19] M. Machholm, E. Lewartowski and C. Courbin, J. Phys. B 27, 4681 (1994).

[20] E. Y. Sidky et al, J. Phys. B 35, 2005 (2002).

[21] In practice, the left, up, right and down contributions to the DCS are obtained by integrating $\sigma_{p_{+1} \rightarrow 3 p}(\theta, \varphi)$ for fixed $\theta$ over the respective quadrants $\varphi=$ $[-\pi / 4, \pi / 4], \quad[\pi / 4,3 \pi / 4], \quad[3 \pi / 4,5 \pi / 4]$ and $[5 \pi / 4,7 \pi / 4]$ (see Fig. 1). This considerably reduces the statistical noise in the measured profiles, but we have verified that the integrated contributions ressemble those obtained for $\varphi=0, \pi / 2, \pi$ and $3 \pi / 2$, respectively.

[22] The values $\alpha_{d}^{N a^{+}}=0.9965$ a.u. and $\alpha_{q}^{N a^{+}}=0.376$ a.u. are taken from [23] while $\alpha_{d}^{R b^{+}}=9.245$ a.u. and $\alpha_{q}^{R b^{+}}=$ 35.41 a.u. are respectively issued from [24] and 25].

[23] J. C. Lombardi, Phys. Rev. A 32, 2569 (1985).

[24] H. Coker, J. Phys. Chem. 80, 2078 (1976).

[25] W. R. Johnson, D. Kolb and K.-N. Huang, At. Data Nucl. Data Tables 28, 334 (1983).

[26] I. V. Hertel et al, Rep. Prog. Phys. 48, 375 (1984).

[27] E. E. B. Campbell, I. V. Hertel and S. E. Nielsen, J. Phys. B 243825 (1991).

[28] S. E. Nielsen and N. Andersen, Z. Phys. D 5, 321 (1987).

[29] J. P. Hansen, S. E. Nielsen and A. Dubois, Phys. Rev. A 46, R5331 (1992). 